\title{
The extended finishing of spiral milling for elliptical inner contour based on FANUC 0i-MB/C/D CNC system
}

\author{
Zhao-Qin Wang ${ }^{1, a^{*}, \text { Xiao-Rong Wang }}{ }^{2, b^{*}}$,Dong-Dong Xie ${ }^{1}$, and Jin-Wu Li ${ }^{1}$ \\ ${ }^{1}$ School of Railway Technology, Lanzhou Jiaotong University, Lanzhou 730010, China \\ ${ }^{2}$ School of Mechatronic Engineering, Lanzhou Jiaotong University, Lanzhou 730010, China \\ awzqpapers@126.com, bwxrlanzhou@qq.com
}

Keywords: extended finishing; spiral milling; ellipse; FANUC; milling

Abstract. Spiral milling is an efficient milling strategy due to reducing non-cutting tool paths. After investigating the characteristics of roughing, semi-finishing and finishing, a new machining process named extended finishing is proposed. The extended finishing has powerful flexibility to realize various machining combinations (such as roughing $(\mathrm{R})$, semi-finishing $(\mathrm{S})$, finishing $(\mathrm{F})$, roughing + semi-finishing + finishing (RSF), roughing + finishing (RF) and semi-finishing + finishing (SF) ) from the three machining processes. With the inner contour of an ellipse, the extended finishing of spiral milling is confirmed to be usable and effective.

\section{Introduction}

Parametric programming (PP) is a basic function equipped by many common $\mathrm{CNC}$ systems such as FANUC, Siemens and HNC. PP is a G/M code programming in which axis positions (X,Y, Z,A,etc) and $\mathrm{F} / \mathrm{S}$ functions can be expressed by a parametric expression [1-3]. PP endows programmers with flexible means to program for parts with typical features and curves/surfaces given by formula. Ellipse is a typical feature and always cut via CNC machines. An ellipse is machined via PP in that the FANUC 0i-MB/C/D CNC systems have no special G code for ellipse cutting. Although the finishing of an ellipse based on FANUC is very mature, the spiral milling hasn't been used in the elliptical milling. Furthermore, for a programmer, he/she has to obtain programs for roughing, semi-finishing and finishing by CAM or constant style manual programming (CSMP)[4]. In this work, a new machining method named extend finishing is presented. Extended finishing (EF), with the elliptical inner contour as an example, integrates roughing, semi-finishing, and finishing processes into one program[5].

By means of PP, for a given part, EF can actualize combination-machining such as roughing (R), semi-finishing $(\mathrm{S})$, finishing $(\mathrm{F})$, roughing + semi-finishing + finishing (RSF), roughing + finishing (RF) and semi-finishing + finishing $(\mathrm{SF})$. Extended finishing has strong industrial application value and prospect for it need no recurring time-consuming and labor-intensive reprogramming like CAM software, and has flexibility of adaptation to new machining conditions that programs from conventional CNC programming and CAM software don't possess, and especially, has the unique ability of selecting combination-machining style online.

\section{The spiral milling of elliptical inner contour}

The standard parametric equation of an ellipse can be expressed as follows:

$$
\left\{\begin{array}{l}
x=a \cos \theta \\
y=b \sin \theta
\end{array}\right.
$$

When spiral milling is used in the milling of the inner contour of an ellipse, the $\mathrm{Z}$ value can be determined as Fig.1 and $\mathrm{Eq}(2)$.

$$
z=\# 5+\frac{\# 7}{360} \theta
$$




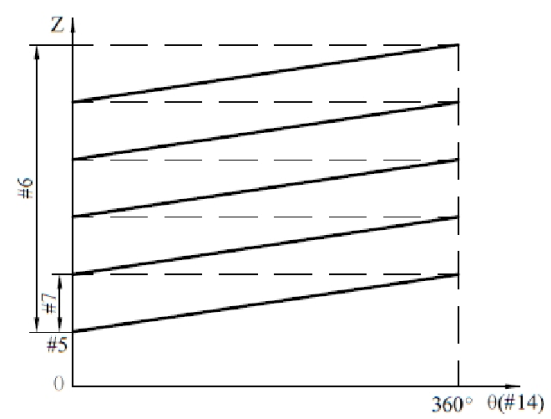

Fig. 1 The $\mathrm{Z}$ value of spiral milling for an elliptical inner contour

\section{The extended finishing method}

In this paper, extended-finishing includes one finishing tool path, several semi-finishing tool paths and several roughing tool paths (as shown in Fig.2). In Fig.2 the radial depths of cut of roughing, semi-finishing and finishing are $\Delta_{r}, \Delta_{s}$, and $\Delta_{f}$ respectively and can be used as parameters and assigned appropriate values manually by MDI keyboard of CNC system. Additionally, $\Delta$ denotes the width of material to be cut, and $n$ is the semi-finishing tool path number, and $m$ is the roughing tool path number.

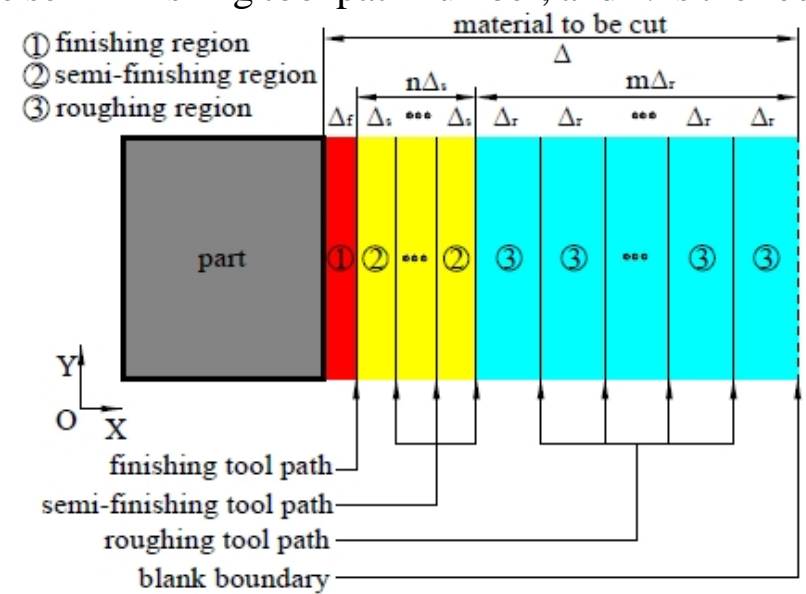

Fig.2 The schematic of extended-finishing

As a result, the subsequent equation expresses the relationship between the width of material to be cut and the radial depths of roughing, semi-finishing and finishing.

$$
\Delta=\Delta_{f}+n \Delta_{s}+m \Delta_{r}
$$

In Eq.(3), $\Delta$ can be known by measuring the blank, and the radial depths of cut $\left(\Delta_{r}, \Delta_{s}\right.$, and $\left.\Delta_{f}\right)$ and the tool path numbers $(n$ and $m$ ) can be determined according to machining process or calculated by parameters.

According to the machining conditions and machining process requirements, the different combinations of the radial depths of cut $\left(\Delta_{r}, \Delta_{s}\right.$, and $\left.\Delta_{f}\right)$ and the tool path numbers $(n$ and $m$ ) corresponding to different extended-finishing pattern are shown in Table 1.

Table1 The extended-finishing pattern

\begin{tabular}{c|l|c|c|c|c|c}
\hline NO. & \multicolumn{1}{|c|}{ Extended-finishing pattern } & $\Delta_{f}$ & $n$ & $\Delta_{s}$ & $m$ & $\Delta_{r}$ \\
\hline 1 & Roughing + semi-finishing + finishing (RSF) & $\bullet$ & $\bullet$ & $\bullet$ & $\circ$ & $\bullet$ \\
\hline 2 & Roughing + finishing (RF) & $\bullet$ & 0 & $\odot$ & $\circ$ & $\bullet$ \\
\hline 3 & Semi-finishing + finishing (SF) & $\bullet$ & $\circ$ & $\bullet$ & 0 & $\odot$ \\
\hline 4 & Roughing (R) & $\odot$ & 0 & $\odot$ & $\circ$ & $\bullet$ \\
\hline 5 & semi-finishing (S) & $\odot$ & $\circ$ & $\bullet$ & 0 & $\odot$ \\
\hline 6 & Finishing (F) & $\bullet$ & 0 & $\odot$ & 0 & $\odot$ \\
\hline
\end{tabular}

Note: • ---given known variable; $\odot$--- given known variable used to calculate;

$\bigcirc$--- variable to be calculate; $\odot$--- random value; 


\section{The spiral milling of extended finishing for elliptical inner contour}

Based on the spiral milling model of ellipses and the concept of extended finishing, the parametric program of an elliptical inner contour using spiral milling of extended finishing is listed as follows:

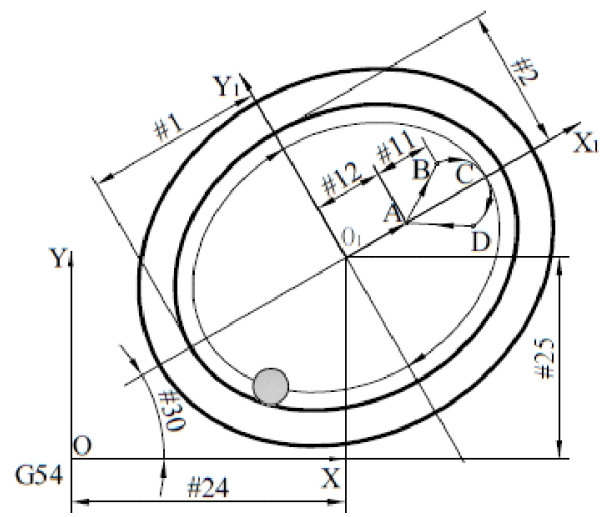

Fig.3 The schematic of tool path of the extended finishing of spiral milling for an elliptical inner contour

Program

Note

$\%$

\section{O5000}

N100 G54G40G49G80G15G17G69

$\mathrm{N} 102 \# 100=1000$

N104 \#101=1

N106 \#102=1

N108 G28G91Z0

N110 G90G53G01Z100H01F3000

N112 M03S3000

N114 M08

$\mathrm{N} 116 \# 1=60$

$\mathrm{N} 118 \# 2=45$

$\mathrm{N} 120 \# 3=13000+\# 102$

N122 \#4=\#[\#3]

$\mathrm{N} 124 \# 5=0$

$\mathrm{N} 126 \# 6=20$

$\mathrm{N} 128 \# 7=5$

$\mathrm{N} 130 \# 8=4$

$\mathrm{N} 132 \# 9=\# 8 * \# 4$

$\mathrm{N} 134 \# 10=4$

$\mathrm{N} 136$ \#11=\#10*\#4

N138 \#12=\#1-\#9-\#11

$\mathrm{N} 140 \# 13=9$

$\mathrm{N} 142 \# 14=1.9$

N144 \#15=\#4+\#13

$\mathrm{N} 146 \# 23=5$

$\mathrm{N} 148$ \#24=0

$\mathrm{N} 150 \# 25=0$

$\mathrm{N} 152 \# 30=45$

N154 IF[\#15EQ\#4]THEN\#15=\#4+0.1

N156 Z[\#5+20]

N158 G52X\#24Y\#25

N160 G68X0Y0R\#30
Initialize

The feedrate base

The feedrate coefficient

The cutter number

$\mathrm{Z}$ homing

Approach to workpiece

Turn spindle on forward

Coolant on

The $\mathrm{X}$-semiaxis of an ellipse

The Y-semiaxis of an ellipse

The system varible for \#102 cutter

Cutter real radius

$\mathrm{Z}$ coordinate (initial value: 0 )

The total height to be cut

$\mathrm{dZ}$

Radius coefficient of $1 / 4$ circle for engage/retract

Radius of 1/4 circle for engage/retract

Projection coefficient of $\mathrm{AB} / \mathrm{AD}$ on $\mathrm{X}$-axis

Projection of $\mathrm{AB} / \mathrm{CD}$ on $\mathrm{X}$-axis

The $\mathrm{X}_{1}$ coordinate of A point per layer

Single side residual thickness

Cutting thichness every time

The setting value of cutter radius

$d \theta$, angle increment of ellipse milling

The $\mathrm{X}$-corrodinate of $\mathrm{O}_{1}$ point in $\mathrm{G} 54$ frame

The Y-corrodinate of $\mathrm{O}_{1}$ point in $\mathrm{G} 54$ frame

As illustrated in Fig.3 


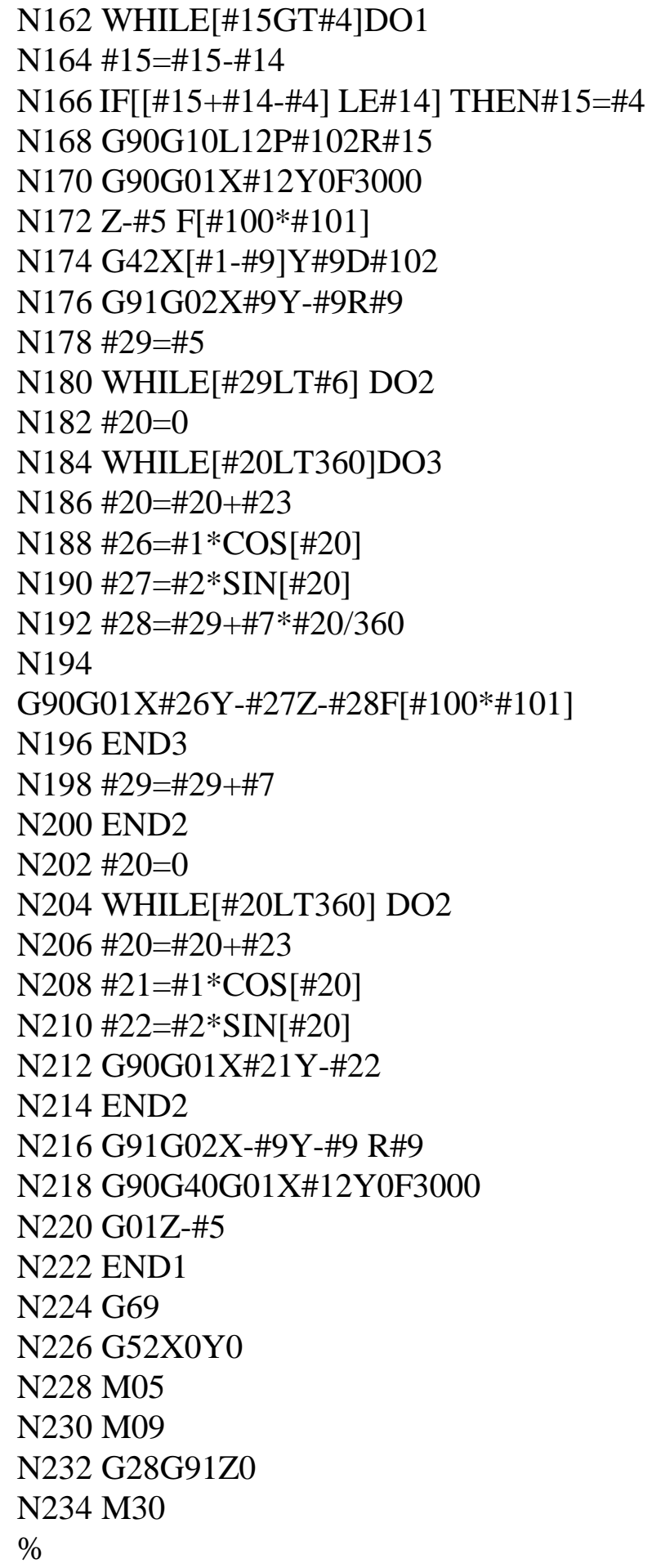

Through changing the values of the relative varibles as listed above, the program of O5000 can perform various combinations of processing method. Especially, the roughing+finishing and finishing results of the inner contour of an ellipse are shown in Fig.4.

\section{Summary}

A novel machining process, extended finishing, is presented. Extended finishing integrates three machining processes of roughing, semi-finishing and finishing into one parametric program. Through altering relative variables, extended finishing can combine into six machining methods (roughing (R), semi-finishing $(\mathrm{S})$, finishing $(\mathrm{F})$, roughing + semi-finishing + finishing (RSF), roughing + finishing (RF) and semi-finishing + finishing $(\mathrm{SF})$ ). With the inner contour of an ellipse, combining spiral milling strategy, the extended finishing of spiral milling is confirmed to be usable and effective. 


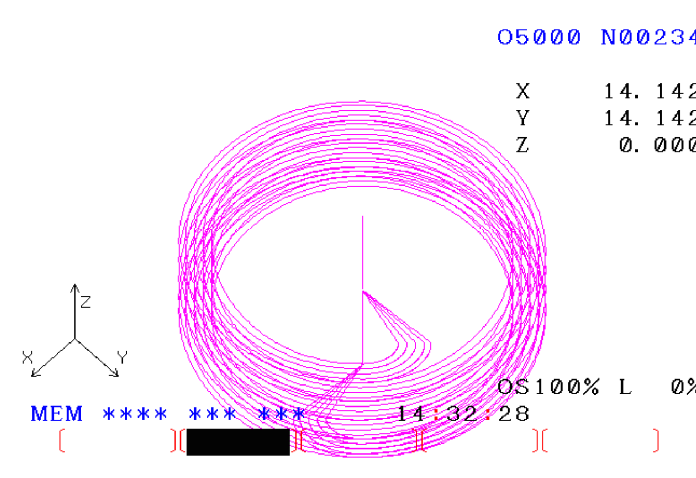

a)3D view(\#13=9)

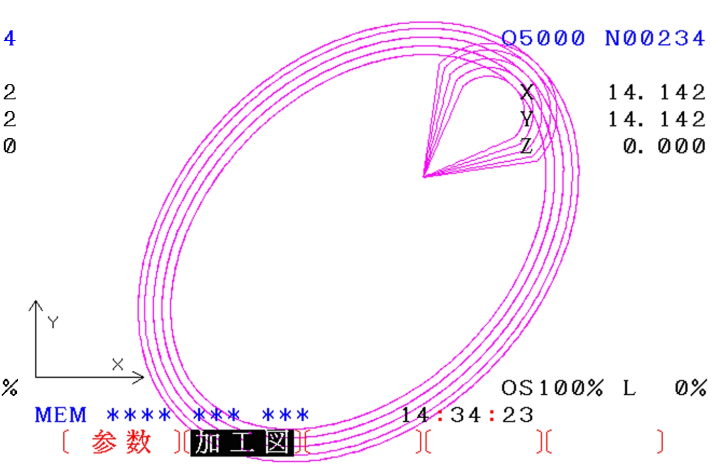

b) XY view(\#13=9)

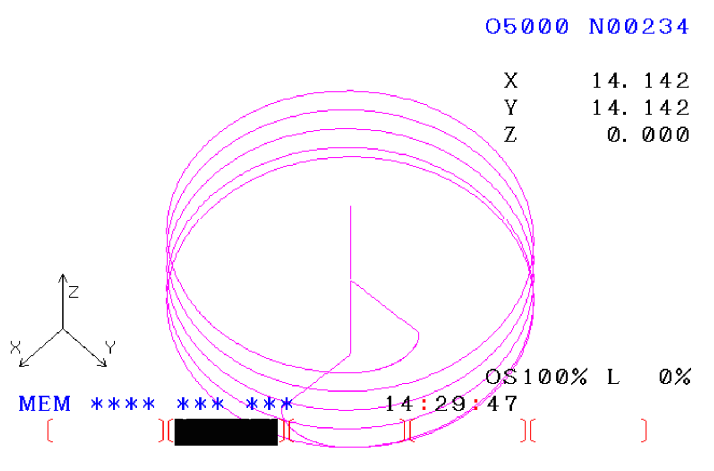

c) $3 \mathrm{D}$ view $(\# 13=0)$

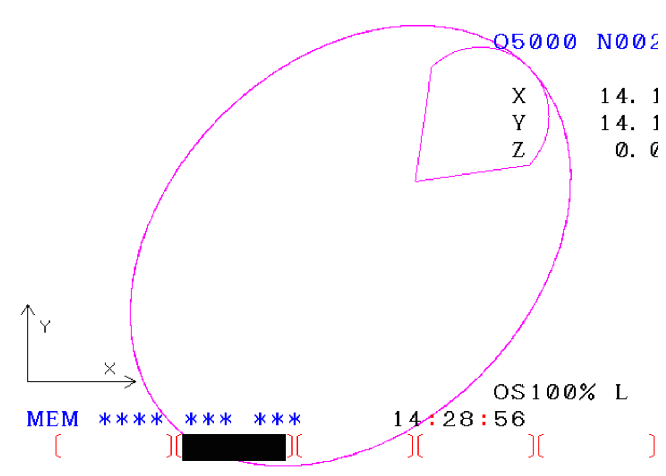

d) $\mathrm{XY} \operatorname{view}(\# 13=0)$

Fig.4 The results of various cutting method combinations of O5000 program

\section{Acknowledgements}

This project is supported by National Natural Science Foundation of China (Grant no. 51465030), Scientific Research Fund of Gansu Provincial Education Department (Grand no. 2015A-050), Natural Science Foundation of Gansu Province (Grant no. 148RJZA035, 148RJYA010).

\section{References}

[1] Z. Wang, X. Wang, The principle and application of cutting-point virtual tool radius compensation for ellipsoidal outer contour finishing using a ball end mill, Int. J. Adv. Manuf. Tech. 71(2014) 1527-1537.

[2] M. Djassemi, A parametric programming technique for efficient CNC machining operations, Comput. Ind. Eng. 35(1998) 33-36.

[3] M. Djassemi, An efficient CNC programming approach based on group technology, J.Manuf. Sys. 19(2000) 213-217.

[4] V. H. C, Learning CAD CAM and CNC machining through mini-car and catapult projects, Int. J. Eng. Edu. 20(2004) 726-732.

[5] Y.-S. Lee, B. Koc, Ellipse-offset approach and inclined zig-zag method for multi-axis roughing of ruled surface pockets, Comput. Aided. Design., 30(1998) 957-971. 